\title{
BMJ Open Epley manoeuvre for posterior semicircular canal benign paroxysmal positional vertigo in people with multiple sclerosis: protocol of a randomised controlled trial
}

\author{
Cristina García-Muñoz (D) ,' María-Dolores Cortés-Vega (D) ,' \\ Juan Carlos Hernández-Rodríguez (1) ,2 Rocio Palomo-Carrión, ${ }^{3}$ \\ Rocío Martín-Valero (D) , ${ }^{4}$ María Jesús Casuso-Holgado (1) ${ }^{1}$
}

To cite: García-Muñoz C, Cortés-Vega M-D, HernándezRodríguez JC, et al. Epley manoeuvre for posterior semicircular canal benign paroxysmal positional vertigo in people with multiple sclerosis: protocol of a randomised controlled trial. BMJ Open 2021;11:e046510. doi:10.1136/ bmjopen-2020-046510

- Prepublication history and additional material for this paper are available online. To view these files, please visit the journal online (http://dx.doi. org/10.1136/bmjopen-2020046510).

Received 02 November 2020 Revised 01 March 2021 Accepted 02 March 2021
Check for updates

(C) Author(s) (or their employer(s)) 2021. Re-use permitted under CC BY-NC. No commercial re-use. See rights and permissions. Published by BMJ.

For numbered affiliations see end of article.

Correspondence to

Dr María-Dolores Cortés-Vega; mdcortes@us.es

\section{ABSTRACT}

Introduction Vestibular disorders in multiple sclerosis (MS) could have central or peripheral origin. Although the central aetiology is the most expected in MS, peripheral damage is also significant in this disease. The most prevalent effect of vestibular peripheral damage is benign paroxysmal positional vertigo (BPPV). Impairments of the posterior semicircular canals represent $60 \%-90 \%$ of cases of BPPV. The standard gold treatment for this syndrome is the Epley manoeuvre (EM), the effectiveness of which has been poorly studied in patients with MS. Only one retrospective research study and a case study have reported encouraging results for EM with regard to resolution of posterior semicircular canal BPPV. The aim of this future randomised controlled trial (RCT) is to assess the effectiveness of EM for BPPV in participants with MS compared with a sham manoeuvre.

Methods and analysis The current protocol describes an RCT with two-arm, parallel-group design. Randomisation, concealed allocation and double-blinding will be conducted to reduce possible bias. Participants and evaluators will be blinded to group allocation. At least 80 participants who meet all eligibility criteria will be recruited. Participants will have the EM or sham manoeuvre performed within the experimental or control group, respectively. The primary outcome of the study is changes in the Dix Hallpike test. The secondary outcome will be changes in self-perceived scales: Dizziness Handicap Inventory and Vestibular Disorders Activities of Daily Living Scale. The sample will be evaluated at baseline, immediately after the intervention and 48 hours postintervention.

Ethics and dissemination The study was approved by the Andalusian Review Board and Ethics Committee of Virgen Macarena-Virgen del Rocio Hospitals (ID 0107-N-20, 23 July 2020). The results of the research will be disseminated by the investigators to peer-reviewed journals.

Trial registration number NCT04578262.

\section{INTRODUCTION}

Multiple sclerosis (MS) is a chronic autoimmune disease characterised by demyelination, gliosis and neural loss. ${ }^{12}$ Disturbances in postural control and dizziness are among

\section{Strengths and limitations of this study}

- This randomised controlled trial will be the first to address knowledge gap on the effectiveness of the Epley manoeuvre in a multiple sclerosis population.

- Double-blind and concealed allocation will reduce the possibility of bias.

- Videonystagmography goggles will enable the primary outcome of the research to be measured more objectively.

- The Epley manoeuvre is an easy and quick vestibular treatment that results in significant changes in benign paroxysmal positional vertigo in a short period of time.

- Due to possible changes in drug treatment and manifestation of multiple sclerosis flares, it is difficult to perform a long-term evaluation of the sample.

the most recurrent symptoms in MS, with a direct repercussion on activities of daily living. ${ }^{3} 4$ This symptomatology could be caused by a vestibular system affection. ${ }^{56}$ In MS, vestibular affection could have peripheral or central origin. ${ }^{78}$ Central vestibulopathy impairments can appear in neuroanatomical locations such as in the eighth cranial nerve, vestibular nuclei, oculomotor tracts, medial longitudinal fasciculus and cerebellum. ${ }^{9}$ On the other hand, peripheral vestibulopathy is due to damages in the vestibular system, highlighting the semicircular canals. ${ }^{5}$ Within these canals, the most common affection is located in the altered information recorded by the posterior semicircular canal. ${ }^{10-12}$ In MS, although central demyelination is the most expected, vestibular peripheral aetiology is also quite common. ${ }^{9} 13$ In the study by Zeigelboim et al, ${ }^{13}$ it was determined that $86 \%$ 
of cases of MS with vestibular problems had a peripheral origin.

The main symptom observed when a vestibulopathy exists is vertigo, defined as an instability associated with a rotation sensation of oneself with respect to the environment. The problem is exacerbated by fast head movements and positional changes. ${ }^{14-16}$ Furthermore, the most common cause of vertigo without another associated neurological sign is benign paroxysmal positional vertigo (BPPV). ${ }^{914} \mathrm{BPPV}$ is a syndrome defined as episodes of vertigo that last less than $60 \mathrm{~s}^{17-19}$ The magnitude of BPPV in patients with MS has been shown in the research of Frohman et $a l,{ }^{9}$ where $52 \%$ of the sample presented BPPV. Affection of the posterior semicircular canals is also apparent in $60 \%-90 \%$ of BPPV cases. ${ }^{20} 21$

The Dix Hallpike manoeuvre is considered the gold standard in identifying posterior semicircular canal BPPV. $^{22}{ }^{23}$ If confirmed in this trial, the treatment of choice will be the Epley manoeuvre (EM), ${ }^{22} 24-26$ which has been reported effective in $80 \%-100 \%$ of patients with posterior canal BPPV. ${ }^{27}$

Due to the central or peripheral origin of vertigo in the MS population, an exhaustive neuro-otological evaluation must be carried out for an accurate diagnosis. ${ }^{7} 8$ Nystagmus is one of the key symptoms to make a differential diagnosis. In vertigo of peripheral origin, nystagmus has a counterclockwise or clockwise movement sum to the horizontal direction. ${ }^{189} 290$ In vertigo of central origin, there are characteristic atypical movements with only one direction. Additionally, peripheral nystagmus is fatigable and fades with an inhibiting effect of ocular fixation. ${ }^{6} 928$ Finally, in patients with MS, if the vertigo is of peripheral origin, a specific vestibular rehabilitation programme can be indicated. ${ }^{79}$ This rehabilitation is based on canalith repositioning procedures aimed at removing the otolith debris inside the particular canal. ${ }^{1322} 27$

Notwithstanding the efficacy of EM for treatment of BPPV, this has been poorly studied in the MS population within the scientific literature. Only two previous research studies have investigated this manoeuvre as an intervention for BPPV in patients with MS. The first was a retrospective study conducted by Frohman $e t a l^{28}$ who reported that $100 \%$ of participants with MS with BPPV successfully recovered after receiving EM. The second was a case study conducted by Yoosefinejad and Siravani ${ }^{31}$ of a participant with relapsing-remitting MS who suffered from BPPV. Interventions applied in this research were the Epley and Semont manoeuvres and the BPPV remitted postintervention. In addition, the participant with MS declared more independence in activities of daily living.

As far as we know, the present study will be the first randomised controlled trial (RCT) to evaluate the application of EM in a population with MS diagnosed with BPPV. The purpose of this trial is to assess the effectiveness of EM in improving BPPV symptoms in participants with MS. We hypothesise a higher efficacy of the intervention for dizziness and activities of daily living improvement when compared with a sham manoeuvre.

\section{METHODS AND ANALYSIS}

\section{Study design}

This protocol describes a two-arm, parallel-group design and a double-blind, randomised clinical trial. A prospective study with randomised and concealed allocation will be performed to prevent possible bias. Participants and evaluators will be blinded to group allocation. The RCT will have three evaluations of the sample which will be carried out at baseline, immediately after the intervention and 48 hours later. The study design is described in figure 1 .

This protocol meets the Standard Protocol Items: Recommendations for Interventional Trials. ${ }^{32}$ The RCT will be developed following instructions from the Consolidated Standards of Reporting Trials. ${ }^{33}$ This study has been registered at ClinicalTrials.gov.

\section{Study setting}

The trial will be conducted at the Physical Therapy Department of the University of Sevilla (Spain). The Vithas Nisa Hospital will be the main healthcare institution involved in this research. Inclusion of other healthcare centres in the area is expected.

\section{Participants and recruitment}

Recruitment of participants is expected to start in February 2021 and is estimated to be completed in June 2022. It will be carried out in the healthcare institutions of the participants. To recruit the study sample, the research team will first contact the Multiple Sclerosis Unit of Seville Vithas Nisa Hospital (Spain). Next, patients with MS who meet the eligibility criteria will be phoned and given an explanation regarding the development and conditions of the RCT. After providing oral and written information, the subjects will be free to decide if they wish to participate. After the invitation, participants who desire to be part of the study will sign the written informed consent (see online supplemental material 1 for the informed consent form).

\section{Inclusion criteria}

- Adults of both genders aged 18-65 years old.

- Clinically diagnosed of any MS subtype (relapsingremitting, primary progressive and secondary progressive).

- Expanded Disability Status Scale score ranging from 1 to 5 points.

- Diagnosed with posterior semicircular canal BPPV by an otolaryngologist and a physical therapist expert in vestibular rehabilitation.

\section{Exclusion criteria}

- Changes in MS pharmacotherapy within the last 3 months.

- BPPV treatments such as vestibular sedatives, corticosteroids, morphine and antihistamines, at least 72 hours before the intervention.

- Alcohol consumption in the last 72 hours.

- Severe visual impairments. 


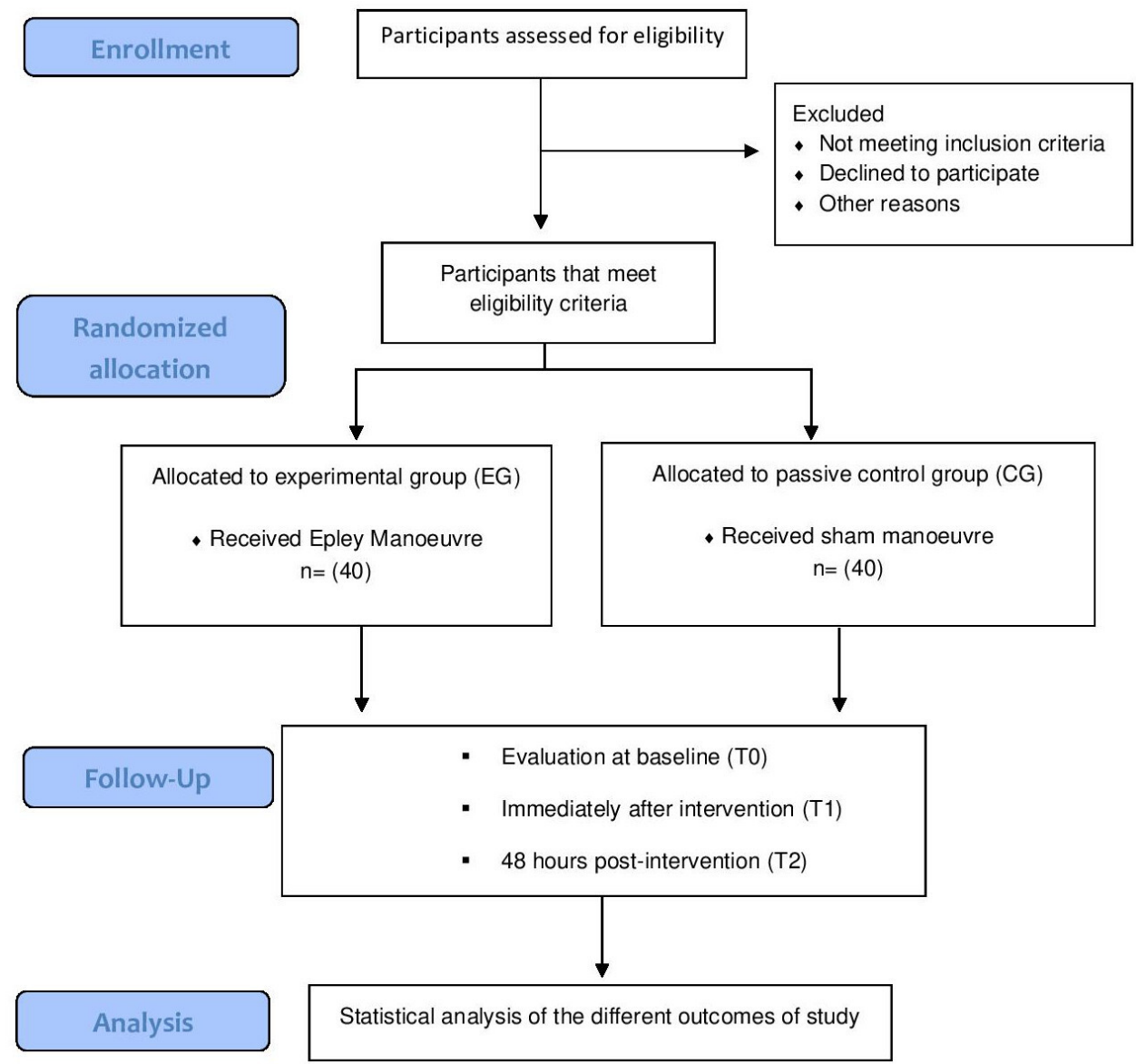

Figure 1 CONSORT flow diagram of participants' recruitment and progress through the phases of the trial. CONSORT, Consolidated Standards of Reporting Trials; CG, control group; EC, experimental group.

- Received vestibular rehabilitation within the last 3 months.

- Existence of any other neurological disease.

\section{Randomisation, concealment allocation and blinding}

Once the informed consent is signed by all participants, a baseline evaluation will be carried out. Randomisation will then be performed by an independent researcher in a computer-generated random sequence. We will consider a 1:1 distribution ratio. The allocation group will be concealed, and it will be sent in an opaque envelope to the physical therapist who will execute the EM. Evaluators and participants will be blinded to group allocation. Double-blinding will reduce the risk of bias.

\section{Patient and public involvement}

Patients or the public are not involved in designing the trial, but a number of public organisations are contacted for patient recruitment (eg, Hospital Virgen Macarena, Ilustre Colegio Profesional de Fisioterapeutas de Andalucía). Once the results are published, participants will be informed by email in an understandable writing. Furthermore, the researchers will perform meetings in each public organisation engaged in the recruitment.
Intervention

Experimental group

Participants allocated to the experimental group will receive the EM, a canalith repositioning procedure. This manoeuvre was developed by Dr John Epley and enables the free-floating otolith debris of the posterior semicircular canal to return to the vestibule..$^{2634} 35$ The subject will undergo only one EM, which will be performed by a physiotherapist expert in vestibular rehabilitation. Before the experimental intervention, all participants will be instructed to keep their eyes open throughout the process. The aim is to detect the appearance of nystagmus in different positions. Moreover, to record and provide quantitative assessment, videonystagmography (VNG) goggles will be worn by participants at the same time as EM is carried out. ${ }^{36}$ The aim of this tool is to record nystagmus along with the EM and to confirm the repositioning of the otoliths after the intervention. ${ }^{37-39} \mathrm{VNG}$ is an infrared camera which detects the black of the pupil, allowing measurement of eye movement speed. ${ }^{38}$ Additionally, thanks to the opaqueness of the VNG, evaluation of nystagmus will not be disturbed by the mechanism of ocular fixation. ${ }^{37}$

EM is a five-step procedure. In the first step, with the patient in supine posture, the head will be positioned 
at $45^{\circ}$ turned towards the unaffected ear, with the head slightly overhanging the edge of the couch. In the second step, maintaining the previous position of the head, the physiotherapist will turn the head $45^{\circ}$ towards the affected ear. In the third step, the whole body will be turned until it is $135^{\circ}$ from the baseline supine position. In the fourth step, while the head is kept turned to the affected ear, the patient will be incorporated until he is sitting. In the fifth step, while the patient is seated with the head in neutral position, the chin will be bent $20^{\circ}$. Each procedure will be held for $30 \mathrm{~s}$ or $2 \mathrm{~min}$ as the dizziness or the nystagmus disappears. After the intervention, the physiotherapist will provide instructions that the participant must follow. The instructions are to not move the head abruptly, to sleep propped up and to not lie down on the affected side in the 48 hours postintervention. ${ }^{35}$ The participant will be re-evaluated 48 hours after the intervention.

\section{Control group}

Participants allocated to the control group will receive a sham manoeuvre. The sham intervention is based on the Semont diagnostic manoeuvre, as described in a study by Bruintjes $e t$ al. $^{35}$ The intervention will also be carried out by an expert physiotherapist. The sham manoeuvre will start with the participant in a neutral seated position. To sum up, the head is rotated $45^{\circ}$ towards the unaffected vestibule, after which the participant will be guided by the physiotherapist to a lateral decubitus position towards the affected side on which his nose will be pointing above. To conclude, the participant will return to a seated position without rotating the head. Each position of the sham manoeuvre will be maintained for $1 \mathrm{~min}$. During the process, the VNG goggles will be worn by the participant and they will be instructed to not close their eyes during the intervention. This group will also be evaluated 48 hours after execution of the manoeuvre.

Once postintervention data are recorded in both groups, EM will be administered to the control group. The same postintervention instructions given to the other group will be followed.

\section{Outcomes and measurements}

The primary outcome of this research will be change from a positive to a negative Dix Hallpike test. The secondary outcome will be self-perceived changes in dizziness symptoms and impact on activities of daily living postintervention.

\section{Change from a positive to a negative Dix Hallpike test}

The Dix Hallpike manoeuvre is targeted to diagnose posterior semicircular canal $\mathrm{BPPV}^{40-42}$ With regard to symptoms, it is necessary to focus on the nystagmus to detect the presence of paroxysmal nystagmus and vertigo. ${ }^{18}{ }^{29}$ In BPPV, nystagmus is defined by fatigability with a duration of under $60 \mathrm{~s}$ and a torsional upbeat direction. ${ }^{43}$ The performance of Dix Hallpike test consists of a subject sitting on a table with the head being turned $45^{\circ}$ towards the side being tested. Once this position is established, the evaluator is going to lay back the patient in a quick movement to produce a neck extension of $20^{\circ}$ while his affected ear down. ${ }^{923}$ Throughout the process, the subject will be instructed to keep their eyes open so that nystagmus can be detected. If torsional nystagmus or vertigo appears while the head is down, it is indicative of posterior canal BPPV. ${ }^{184}$ In the current protocol, the Dix Hallpike test will be supported by VNG. This tool provides objective information on the Dix Hallpike test, thanks to the pupil tracking software which assesses eye movement and speed. ${ }^{37}$ Testing with VNG provides several advantages, such as evaluation of the eyes by the clinician in real time, recording of video for documentation and later review, and recognition of torsional eye movement. Also, nystagmus will not be disturbed by environment illumination and mechanisms of ocular fixation. ${ }^{3843}$

\section{Dizziness}

Dizziness will be assessed using the Dizziness Handicap Inventory (DHI). The aim of this inventory is to assess the impact of dizziness on the quality of life. In addition, it evaluates the perception of balance in activities of daily living. The DHI is a 25-item self-assessment questionnaire with a total score of 100 . The score is calculated by summing the responses to ordinal scales. A higher score means a higher level of disability and handicap, composed of physical, emotional and functional subscales. Scores for physical and emotional subscales range from 0 to 36 points, and for functional subscale from 0 to 28 points. ${ }^{43} 4546$

\section{Activities of daily living}

The Vestibular Disorders Activities of Daily Living Scale (VADL) is a self-reported questionnaire that measures independence in activities of daily living of people with vestibular disorders. ${ }^{47}$ This scale is composed of 28 items divided into 3 subcategories: 12 questions on functional skills, 9 questions on ambulation skills and 7 questions on instrumental skills. Each item is rated on a 10-point scale, where a higher score means less independence in activities of daily living. The total score is the median of each subscale. ${ }^{48} 49$

Evaluation of the sample will be performed at baseline (T0), immediately after the intervention only for the Dix Hallpike manoeuvre (T1) and 48 hours postintervention (T2). VADL will be again evaluated 1 week postintervention (table 1).

\section{Sample size calculation}

The sample size was calculated using the G*Power software (V.3.1.7; Kiel University, Kiel, Germany). ${ }^{50}$ The sample size has been calculated as 80 participants for a one-tailed hypothesis, with an $\alpha$ error of 0.05 , size effect of $\mathrm{d}=0.8$, statistical power of $80 \%$ and an estimated dropout rate of $15 \% .{ }^{5152}$

\section{Statistical analysis}

Normal distribution of variables will be assessed by Shapiro-Wilk test, and the Levene test will be carried out for variance homogeneity. Regarding the 
description of quantitative variables, it will be drawn on central tendency measures and dispersion as mean and SD when they follow normal distribution. On the other hand, when variables do not follow this distribution, median, minimum and maximum intervals, and percentiles will be shown. Additionally, the results for the qualitative variables will be shown as absolute and relative frequencies.

For normal distribution, Student's t-test will be implemented to compare the means of independent samples. On the other hand, similar non-parametric tests will be applied in case of non-normal distribution. Cohen's criteria will be followed to assess the effect size of the studied variables. A $95 \%$ CI will be considered. Intention-to-treat principles will be considered for all analyses. Graphical and numerical analyses of data will be conducted using SPSS V.25.0 and GraphPad Prism (GraphPad, San Diego, California, USA).

\section{Ethics and dissemination}

The study was approved by the Andalusian Review Board and Ethics Committee of Virgen Macarena-Virgen del Rocio Hospitals (ID 0107-N-20, 23 July 2020). All participants will undergo and accept informed consent before data compilation. The investigators will disseminate the study results via publication in peer-reviewed scientific journals.

\section{DISCUSSION}

The aim of this future RCT is to assess the effectiveness of a vestibular rehabilitation intervention based on EM in participants with MS with posterior semicircular canal BPPV.

Vestibular dysfunction in MS can be caused by a central demyelination of the vestibular tracts and a peripheral vestibular damage, or both at the same time. These affections may induce an incorrect vestibular ocular or vestibulospinal reflex response, resulting in ocular and balance disorders. ${ }^{7} 132853$ Imbalance and vertigo are symptoms used to be misdiagnosed by healthcare professionals especially in people with MS. ${ }^{54}$ This is due to the myriad of aetiologies of vestibular impairments in $\mathrm{MS}^{13}$ In most cases, central vestibulopathy in MS is taken as the main cause of vertigo even without accurate neuro-otological examination. ${ }^{79}$ However, peripheral vestibular disorders are quite recurrent in the MS population, with BPPV being the most common. ${ }^{7}$ Although the posterior semicircular canal is mainly affected in BPPV, we should also remember that this canal could be damaged by several causes that also result in vertigo. Because of this, an accurate diagnosis of posterior semicircular canal impairment is necessary. One of the possible affections of the inner ear is the posterior semicircular canal dehiscence, caused by a defect in the bony roof of the temporal bone. Thus, Thabet $e t a l^{55}$ state that contralateral oVemps (extraocular vestibular-evoked myogenic potential) response and multidetector CT scanner (MDCTS) are essential to rule out inner ear defects and to reach a correct diagnosis. Reaching a correct vestibular diagnosis in MS is difficult, which is why diagnostic tools such as VNG, electronystagmography, cervical or ocular Vemps, magnetic resonance, MDCTS and specific vestibular manoeuvres, along with clinical manifestations, could play a fundamental role in the neuro-otological examination. ${ }^{95-60}$

Due to misdiagnosis, people with MS who suffer from acute BPPV are improperly treated because they receive pharmacological treatment instead of choice therapy. An elective treatment prevents patients with MS from unnecessary ingestion of drugs, including vestibular sedatives, corticosteroids, morphine and antihistamines. ${ }^{142}$ The proper approach in BPPV is the EM. ${ }^{17} 17634$ In this future RCT, to avoid misdiagnosis and to recruit subjects with MS with BPPV, an extensive neuro-otological evaluation will be carried out. ${ }^{5861}$ 
EM for resolution of posterior semicircular canal BPPV has been studied in depth in patients having only this syndrome. ${ }^{21} 2734$ The success of EM has also been proven against other canalith repositioning procedures by Brandt-Daroff. ${ }^{62}$ Furthermore, Bruintjes $e t a l^{35}$ revealed that EM has long-term effects after the intervention. The impact of dizziness on activities of daily living was also assessed. In this case, significant changes were recorded for DHI after an intervention based on EM. ${ }^{35}$ The results of the study by Gupta $e t a l^{25}$ showed that canalith repositioning procedures of EM are suitable in improving the quality of life of people who suffer from BPPV.

Even though research has investigated patients with BPPV only, the effectivity of EM in MS has not been studied sufficiently. The previously mentioned studies conducted by Frohman et $a l^{28}$ and Yoosefinejad and Siravani ${ }^{31}$ are the only ones that performed EM as an intervention. Several studies have shown that the MS population with BPPV would benefit from specific rehabilitation programmes based on EM. They also exposed the need for scientific papers to fill the knowledge gap on this topic in this patient population. ${ }^{2728}$ Therefore, to improve the strength of evidence, the current protocol describes a prospective double-blind RCT that will carry out EM in patients with MS with BPPV. If the intervention proves to be effective, its outcome could reduce unnecessary pharmacological treatments and therefore healthcare expenditure. Furthermore, if dizziness is reduced in MS, a lower self-perceived disease impact on activities of daily living could be achieved.

\section{Author affiliations \\ ${ }^{1}$ Department of Physiotherapy, University of Seville, Sevilla, Spain \\ ${ }^{2}$ Dermatology Department, Hospital Universitario Virgen del Rocío, Sevilla, Spain \\ ${ }^{3}$ Department of Nursery, Physiotherapy and Occupational Therapy, Universidad de Castilla-La Mancha, Toledo, Spain \\ ${ }^{4}$ Department of Physiotherapy, University of Malaga, Malaga, Spain}

Contributors CG-M, M-DC-V and M-JC-H conceptualised and designed the study. CG-M wrote the first draft of the manuscript with critical input from M-JC-H and MDC-V. M-DC-V, M-JC-H, JCH-R, RP-C and RMV contributed significantly to revision of the manuscript. All authors read and approved the final manuscript.

Funding The authors have not declared a specific grant for this research from any funding agency in the public, commercial or not-for-profit sectors.

Competing interests None declared.

Patient consent for publication Not required.

Provenance and peer review Not commissioned; externally peer reviewed.

Supplemental material This content has been supplied by the author(s). It has not been vetted by BMJ Publishing Group Limited (BMJ) and may not have been peer-reviewed. Any opinions or recommendations discussed are solely those of the author(s) and are not endorsed by BMJ. BMJ disclaims all liability and responsibility arising from any reliance placed on the content. Where the content includes any translated material, BMJ does not warrant the accuracy and reliability of the translations (including but not limited to local regulations, clinical guidelines, terminology, drug names and drug dosages), and is not responsible for any error and/or omissions arising from translation and adaptation or otherwise.

Open access This is an open access article distributed in accordance with the Creative Commons Attribution Non Commercial (CC BY-NC 4.0) license, which permits others to distribute, remix, adapt, build upon this work non-commercially, and license their derivative works on different terms, provided the original work is properly cited, appropriate credit is given, any changes made indicated, and the use is non-commercial. See: http://creativecommons.org/licenses/by-nc/4.0/.

\section{ORCID iDs}

Cristina García-Muñoz http://orcid.org/0000-0003-2621-2098

María-Dolores Cortés-Vega http://orcid.org/0000-0002-9514-8811

Juan Carlos Hernández-Rodríguez http://orcid.org/

0000-0003-2525-40690000-0003-2525-4069

Rocío Martín-Valero http://orcid.org/0000-0002-1664-3647

María Jesús Casuso-Holgado http://orcid.org/0000-0002-4217-6827

\section{REFERENCES}

1 Tafti D, Ehsan M, Xixis KL. Multiple Sclerosis. StatPearls Publishing, 2020.

2 Wallin MT, Culpepper WJ, Nichols E, et al. Global, regional, and national burden of multiple sclerosis 1990-2016: a systematic analysis for the global burden of disease study 2016. Lancet Neurol 2019;18:269-85.

3 Gossman W, Ehsan M, Xixis KL. Multiple Sclerosis, 2019.

4 Marrie RA, Cutter GR, Tyry T. Substantial burden of dizziness in multiple sclerosis. Mult Scler Relat Disord 2013;2:21-8.

5 Kutz JW. The dizzy patient. Med Clin North Am 2010;94:989-1002.

6 Doty RL, MacGillivray MR, Talab H, et al. Balance in multiple sclerosis: relationship to central brain regions. Exp Brain Res 2018;236:2739-50.

7 Alpini D, Caputo D, Pugnetti L, et al. Vertigo and multiple sclerosis: aspects of differential diagnosis. Neurol Sci 2001;22 Suppl 2:S84-7.

8 Alpini D, Berardino FD, Mattei V, et al. Characteristics of multiple sclerosis patient stance control disorders, measured by means of posturography and related to brainstem lesions. Audiol Res 2012;2:43-7.

9 Frohman EM, Kramer PD, Dewey RB, et al. Benign paroxysmal positioning vertigo in multiple sclerosis: diagnosis, pathophysiology and therapeutic techniques. Mult Scler 2003;9:250-5.

10 Soto-Varela A, Santos-Perez S, Rossi-Izquierdo M, et al. Are the three canals equally susceptible to benign paroxysmal positional vertigo? Audiol Neurootol 2013;18:327-34.

11 Instrum RS, Parnes LS. Benign paroxysmal positional vertigo. Adv Otorhinolaryngol 2019;82:67-76.

12 Balatsouras DG. Benign paroxysmal positional vertigo with multiple canal involvement. Am J Otolaryngol 2012;33:250-8.

13 Zeigelboim BS, Arruda WO, Mangabeira-Albernaz PL, et al. Vestibular findings in relapsing, remitting multiple sclerosis: a study of thirty patients. Int Tinnitus J 2008;14:139-45.

14 Frohman TC, O' donghue DL, Northrop D. Multiple Sclerosis for the Physician Assistant. In: Multiple Sclerosis for the Physician Assistant. National Multiple Sclerosis Society 2011:138-41.

15 Song MH, Kong TH, Shim DB. Optimal reassessment time for treatment response in posterior canal benign paroxysmal positional vertigo. Laryngoscope 2019:1-4.

16 Kollén L, Bjerlemo B, Möller C. Evaluation of treatment in benign paroxysmal positional vertigo (BPPV). Adv Physiother 2006;8:106-15.

17 Lindell E, Kollén L, Johansson M. Benign paroxysmal positional vertigo, dizziness, and health-related quality of life among older adults in a population-based setting. Eur Arch Oto-RhinoLaryngology.

18 Bhattacharyya N, Gubbels SP, Schwartz SR, et al. Clinical practice guideline: benign paroxysmal positional vertigo (update). Otolaryngol Head Neck Surg 2017;156:S1-47.

19 Strupp M, Mandalà M, López-Escámez JA. Peripheral vestibular disorders: an update. Curr Opin Neurol 2019;32:165-73.

20 Ciorba A, Cogliandolo C, Bianchini C, et al. Clinical features of benign paroxysmal positional vertigo of the posterior semicircular canal. SAGE Open Med 2019;7:205031211882292.

21 Ballvé JL, Carrillo-Muñoz R, Rando-Matos Y, et al. Effectiveness of the Epley manoeuvre in posterior canal benign paroxysmal positional vertigo: a randomised clinical trial in primary care. $\mathrm{Br} J$ Gen Pract 2019;69:e52-60.

22 Mandalà M, Salerni L, Nuti D. Benign positional paroxysmal vertigo treatment: a practical update. Curr Treat Options Neurol 2019;21:66.

23 Dix MR, Hallpike S, Meniere P. The pathology, symptomatology and diagnosis of certain common disorders of the vestibular system, 1952.

24 Lin HC, Shu MT, Shi SM. The canalith repositioning procedure for benign paroxysmal positional vertigo. J Taiwan Otolaryngol - Head Neck Surg 1999;34:379-84.

25 Gupta AK, Sharma KG, Sharma P. Effect of Epley, Semont maneuvers and Brandt-Daroff exercise on quality of life in patients 
with posterior semicircular canal benign paroxysmal positional vertigo (PSCBPPV). Indian J Otolaryngol Head Neck Surg 2019;71:99-103.

26 Epley JM. The canalith repositioning procedure: for treatment of benign paroxysmal positional vertigo. Otolaryngol Head Neck Surg 1992;107:399-404.

27 Hunt WT, Zimmermann EF, Hilton MP. Modifications of the Epley (canalith repositioning) manoeuvre for posterior canal benign paroxysmal positional vertigo (BPPV). Cochrane Database Syst Rev 2012; Published Online First.

28 Frohman EM, Zhang H, Dewey RB, et al. Vertigo in MS: utility of positional and particle repositioning maneuvers. Neurology 2000;55:1566-9.

29 Ling X, Zhao D-H, Shen B, et al. Clinical characteristics of patients with benign paroxysmal positional vertigo diagnosed based on the diagnostic criteria of the Bárány Society. Front Neurol 2020;11.

30 Power L, Murray K, Szmulewicz DJ. Characteristics of assessment and treatment in benign paroxysmal positional vertigo (BPPV). VES 2020;30:55-62.

31 Yoosefinejad AK, Siravani F. Semont and Epley maneuvers alleviated vertigo in a patient with multiple sclerosis. Heal Sci J 2015;9:1-3.

32 Chan A-W, Tetzlaff JM, Altman DG, et al. Spirit 2013 statement: defining standard protocol items for clinical trials. Ann Intern Med 2013;158:200-7.

33 Schulz KF, Altman DG, CONSORT MD. Statement: updated guidelines for reporting parallel group randomized trials. Ann Intern Med 2010;2010:726-32.

34 Hilton MP, Pinder DK. The Epley (canalith repositioning) manoeuvre for benign paroxysmal positional vertigo. Cochrane Database Syst Rev 2014;2014:CD003162.

35 Bruintjes TD, Companjen J, van der Zaag-Loonen HJ, et al. A randomised sham-controlled trial to assess the long-term effect of the Epley manoeuvre for treatment of posterior canal benign paroxysmal positional vertigo. Clin Otolaryngol 2014;39:39-44.

36 Maslovara S, Vešligaj T, Soldo SB. Importance of accurate diagnosis in benign paroxysmal positional vertigo (BPPV) therapy. Med Glas 2014;11:300-6.

37 VanDerHeyden CM, Carender WJ, Heidenreich KD. Nystagmus discordance with 2-dimensional videonystagmography in posterior semicircular canal benign paroxysmal positional vertigo. Otolaryngol Head Neck Surg 2015;152:724-8.

38 Falls C. Videonystagmography and posturography. Adv Otorhinolaryngol 2019;82:32-8.

39 Shah S, Vishwakarma R. Role of Videonystagmography (VNG) in Epley's Maneuver. Int J Otolaryngol Head 2014;03:311-7.

40 Kinne BL. Benign paroxysmal positional vertigo eye Mov Disord (nystagmus strabismus) diagnosis. Manag Impact Qual Life 2014:1-30.

41 Rizk H, Agrawal Y, Barthel S, et al. Quality improvement in neurology: neuro-otology quality measurement set. Neurology 2018;91:697-701.

42 Dunlap PM, Holmberg JM, Whitney SL. Vestibular rehabilitation: advances in peripheral and central vestibular disorders. Curr Opin Neurol 2019;32:137-44.

43 Martens C, Goplen FK, Aasen T, et al. Dizziness handicap and clinical characteristics of posterior and lateral canal BPPV. Eur Arch Otorhinolaryngol 2019;276:2181-9.

44 Domingues R, Waseem M. Dix-Hallpike Maneuver. In: Atlas of emergency medicine procedures. Springer New York, 2016: 257-8.
45 Tamber A-L, Wilhelmsen KT, Strand LI. Measurement properties of the dizziness handicap inventory by cross-sectional and longitudinal designs. Health Qual Life Outcomes 2009;7:1-16.

46 Cattaneo D, Jonsdottir J, Repetti S. Reliability of four scales on balance disorders in persons with multiple sclerosis. Disabil Rehabil 2007;29:1920-5.

47 Harun A, Semenov YR, Agrawal Y. Vestibular function and activities of daily living. Gerontology and Geriatric Medicine 2015;1:233372141560712.

48 Cohen HS. Use of the vestibular disorders activities of daily living scale to describe functional limitations in patients with vestibular disorders. J Vestib Res 2014;24:33-8.

49 Cohen HS, Kimball KT, Adams AS. Application of the vestibular disorders activities of daily living scale. Laryngoscope 2000;110:1204-9.

50 Faul F, Erdfelder E, Lang AG. G*Power 3: A flexible statistical power analysis program for the social, behavioral, and biomedical sciences. In: Behavior Research Methods. Psychonomic Society Inc 2007:175-91.

51 Kieser M. Methods and applications of sample size calculation and Recalculation in clinical trials. Springer.

52 Verma JP, Verma P. Determining sample size and power in research studies. Springer Singapore, 2020.

53 Marsden J, Pavlou M, Dennett R. Vestibular rehabilitation in multiple sclerosis: study protocol for a randomised controlled trial and cost-effectiveness analysis comparing customised with booklet based vestibular rehabilitation for vestibulopathy and a 12 month observational cohort stud. BMC Neurol 2020;20:1-15.

54 Thomas DB, Newman-Toker DE. Diagnosis is a team sport partnering with allied health professionals to reduce diagnostic errors. Diagnosis 2016;3:49-59.

55 Thabet EM, Abdelkhalek A, Zaghloul H. Superior semicircular canal dehiscence syndrome as assessed by oVEMP and temporal bone computed tomography imaging. Eur Arch Otorhinolaryngol 2012;269:1545-9.

56 Di Stadio A, Dipietro L, Ralli M, et al. The role of vestibular evoked myogenic potentials in multiple sclerosis-related vertigo. A systematic review of the literature. Mult Scler Relat Disord 2019;28:159-64.

57 Korres S, Riga M, Papacharalampous G, et al. Relative diagnostic importance of electronystagmography and magnetic resonance imaging in vestibular disorders. J Laryngol Otol 2009;123:851-6.

58 Esra E, Mecidiyekoy O, Hastanesi C. Importance of accurate diagnosis in benign paroxysmal positional vertigo with Videonstagmography and the success rate of the maneuvers, 2017.

59 Mohamed ES. Predictors of central vestibular disorders from videonystagmography tests. Egypt J Otolaryngol 2016;32:202-9.

60 Woo D, Frohman TC, Frohman EM. Vestibular testing and multiple sclerosis. Handb Clin Neurophysiol 2010;9:478-86.

61 Di Stadio A, Ralli M. Inner ear involvement in multiple sclerosis: an underestimated condition? Mult Scler 2018;24:1264-5.

62 Cetin YS, Ozmen OA, Demir UL, et al. Comparison of the effectiveness of Brandt-Daroff vestibular training and Epley canalith repositioning maneuver in benign paroxysmal positional vertigo long term result: a randomized prospective clinical trial. Pak J Med Sci 2018;34:558-63. 
Correction: Epley manoeuvre for posterior semicircular canal benign paroxysmal positional vertigo in people with multiple sclerosis: protocol of a randomised controlled trial

García-Muñoz C, Cortes-Vega MD, Hernández-Rodríguez JC, et al. Epley manoeuvre for posterior semicircular canal benign paroxysmal positional vertigo in people with multiple sclerosis: protocol of a randomised controlled trial BMJOpen 2021;11:e046510. doi: 10.1136/bmjopen-2020-046510

This article was previously published with errors. Author names have been updated with the correct hyphenations.

Open access This is an open access article distributed in accordance with the Creative Commons Attribution Non Commercial (CC BY-NC 4.0) license, which permits others to distribute, remix, adapt, build upon this work non-commercially, and license their derivative works on different terms, provided the original work is properly cited, appropriate credit is given, any changes made indicated, and the use is non-commercial. See: http://creativecommons.org/licenses/by-nc/4.0/.

(c) Author(s) (or their employer(s)) 2021. Re-use permitted under CC BY-NC. No commercial re-use. See rights and permissions. Published by BMJ.

BMJ Open 2021;11:e046510corr1. doi:10.1136/bmjopen-2020-046510corr1

D) Check for updates 